\title{
Oxidative and Antioxidative Stress Linked Biomarkers in Ankylosing Spondylitis: A Systematic Review and Meta-analysis
}

\author{
Jiaxiao Li, ${ }^{1,2}$ Suling Liu, ${ }^{2,3}$ and Yang Cui $\mathbb{i D}^{1,2,3}$ \\ ${ }^{1}$ South China University of Technology, Guangzhou, Guangdong Province, China \\ ${ }^{2}$ Department of Rheumatology and Immunology, Guangdong Provincial People's Hospital, Guangdong Academy of Medical Sciences, \\ Guangzhou, Guangdong, China \\ ${ }^{3}$ Shantou University Medical College, Shantou, Guangdong Province, China
}

Correspondence should be addressed to Yang Cui; 13602835538@139.com

Received 23 June 2020; Revised 7 September 2020; Accepted 14 September 2020; Published 21 September 2020

Academic Editor: Sid D. Ray

Copyright ( 2020 Jiaxiao Li et al. This is an open access article distributed under the Creative Commons Attribution License, which permits unrestricted use, distribution, and reproduction in any medium, provided the original work is properly cited.

\begin{abstract}
Objective. Ankylosing spondylitis (AS) is a chronic inflammatory disease that affects the axial skeleton, leading to joint disability. Our study aims at investigating the change of oxidative and antioxidative stress linked biomarkers in AS. Methods. This systematic review and meta-analysis was performed following the Preferred Reporting Items of Systematic Reviews and Meta-Analyses (PRISMA) statement. PubMed, Web of Science, and Cochrane Library databases till May 2020 were searched. Only articles published in English were included. Two reviewers screened relevant studies, extracted data, and assessed the quality of included studies using the Newcastle-Ottawa scale independently. Either random or fixed effect model was adopted base on the heterogeneity testing by $I^{2}$ statistic. Standardized mean difference (SMD) and 95\% confidence intervals (CI) of oxidative and antioxidative markers were calculated. $p$ value $<0.05$ was considered statistically significant. Results. A total of 22 studies including 931 AS patients and 917 healthy controls met the selection criteria. Significantly increased levels of all oxidative stress markers except myeloperoxidase, and significantly decreased levels of total antioxidant status $(\mathrm{SMD}=-1.19$, $95 \%$ CI -2.35 to $-0.03, p=0.044)$ and paraoxonase $1(\mathrm{SMD}=-1.01,95 \%$ CI -1.78 to $-0.24, p=0.010)$ in serum were observed in AS patients when compared with healthy controls. However, there were no significant differences of all oxidative and anti-oxidative stress biomarkers in erythrocytes. Additionally, the levels of malondialdehyde (SMD $=0.51,95 \%$ CI 0.21 to $0.81, p=0.001$ ) and advanced oxidation protein products (SMD $=0.95,95 \%$ CI 0.58 to $1.31, p<0.001$ ) in serum were significantly higher in active patients when compared with inactive AS patients. Conclusion. This meta-analysis demonstrated an overall increase of oxidative markers and decrease of antioxidative markers in AS, suggesting that oxidative stress may play an important role in the pathogenesis of AS.
\end{abstract}

\section{Introduction}

Ankylosing spondylitis (AS) is the major subtype of spondyloarthropathies involving predominantly the sacroiliac joints and axial skeleton and can lead to impairments in joint structures, new bone formation, and disability [1]. Furthermore, other specific organs and systems such as eyes, skin, kidneys, gastrointestinal tract, and cardiovascular system can be affected with the development of AS, resulting in decreased quality of life [2]. Although the etiology of AS is still unclear, it has been reported that oxidative stress may play an important role in the pathogenesis of AS [3, 4].
Oxidative stress refers to the imbalance of oxidative and antioxidative systems, resulting in an increase of reactive oxygen species (ROS) [5]. ROS including nitric oxide, superoxide, and hydroxyl radical anion can be produced in normal cellular metabolism and is crucial for cell proliferation, differentiation, apoptosis, and signal transduction with a low concentration [6]. However, oxidative stress happens when the speed of the increase of ROS exceeds the capacity of antioxidant to eliminate them, triggering oxidative damage to lipids, proteins, and DNA [5]. Various byproducts would be produced in oxidative damage such as malondialdehyde (MDA) and advanced oxidation protein products (AOPP). 
MDA is a marker of lipid peroxidation [7], which can work as secondary mediators to cause further damage [8]. AOPP, a marker of protein oxidation products with earlier formation and greater stability in the process of oxidative damage, can also demonstrate oxidative stress [3]. On the contrary, antioxidants such as catalase, superoxide dismutase (SOD), and paraoxonase $(\mathrm{PON})$ can protect against oxidative damage [9]. For example, SOD can convert superoxidative radicals to hydrogen peroxide by disproportionation, and then hydrogen peroxide can be reduced to water and oxygen by CAT [10]. Therefore, the concentrations of antioxidants also reflect the oxidative stress state.

Oxidative stress is associated with inflammation, the pathological hallmark of AS [11]. On one hand, inflammatory cells can promote the release of various enzymes to induce oxidative stress [12]. On the other hand, a number of ROS accumulating at the site of inflammation can enhance proinflammatory responses by activating the inflammatory signal pathways [13]. The interconnected relationship between bone inflammation and oxidative stress in AS contributes to bone resorption $[8,14]$. Acting as both cause and effect, inflammatory cytokines and oxidative stress can promote the formation and activation of osteoclast to enhance bone loss in AS [8]. Signal pathways activated by proinflammatory cytokines and oxidative stress products also play a key role in the progression of bone mass loss in AS [14]. In addition, new bone formation is another pathological feature of AS. Although the role of oxidative stress in new bone formation is still unclear, it is reported that ROS is related to the activation of $\mathrm{Wnt} /$ Beta-catenin and $\mathrm{BMP} / \mathrm{Smad}$ pathways which are involved in the progression of new bone formation in AS $[15,16]$.

Up to now, the levels of oxidative and antioxidative biomarkers in serum and erythrocytes of AS patients have been assessed in several studies. However, the results in different studies were inconsistent. Thus, we aimed to investigate the change of oxidative and antioxidative stress linked biomarkers in AS and to explore the potential role of oxidative stress in the pathogenesis of AS. To our knowledge, this is the first meta-analysis of oxidative and antioxidative biomarkers in AS.

\section{Methods}

2.1. Literature Search Strategy. This systematic review and meta-analysis was performed following the Preferred Reporting Items of Systematic Reviews and Meta-Analyses (PRISMA) statement, and the protocol was registered in INPLASY (registration number: INPLASY202050066). PubMed, Web of Science, and Cochrane Library databases till May 2020 were searched following the protocol. The search terms are as follows: ("oxidative" or "oxidation" or "oxidant" or "oxidat*" or "antioxidant" or "antioxidative" or "antioxidat*" or "redox") AND ("spondylitis, ankylosing" or "spondyloarthritis ankylopoietica" or "ankylosing spondylarthritis" or "ankylosing spondylarthritides" or "spondylarthritides, ankylosing" or "spondylarthritis, ankylosing" or "ankylosing spondylitis" or "spondylarthritis ankylopoietica" or "bechterew disease" or "bechterew's disease" or "bechterew s disease" or "marie strum- pell disease" or "marie strumpell disease" or "rheumatoid spondylitis" or "spondylitis, rheumatoid" or "spondylitis ankylopoietica" or "ankylosing spondyloarthritis" or "ankylosing spondylarthritides" or "spondylarthritides, ankylosing" or "spondyloarthritis, ankylosing" or "axial spondyloarthritis" or "peripheral spondyloarthritis" or "radiographic axial spondyloarthritis"). There was no language restriction when searching the above databases. Two independent reviewers searched the above databases and screened the titles and abstracts. In addition, references of searched studies were reviewed to identify additional relevant studies. After that, full texts of relevant studies were reviewed. Any disagreements between two reviewers were resolved by the third author.

2.2. Inclusion and Exclusion Criteria. Studies were included when they met the following criteria: (1) case-control or cohort study, (2) measurement of oxidative or antioxidative stress biomarkers in any type of samples from AS patients and healthy controls, and (3) English language. Exclusion criteria are as follows: (1) reviews, case reports, letters, meeting, and abstracts; (2) insufficient data; and (3) animal or in vitro study.

2.3. Data Extraction. The data of studies meeting the selection criteria were extracted by two authors independently. Any disagreements between two reviewers were resolved by the third author. The following data were extracted: first author, publication year, country of studies, age, gender, sample size and source, type of markers, the concentration of oxidative or antioxidative markers, diagnostic criteria, and disease activity. The Newcastle-Ottawa scale (NOS) was used to assess the quality of included studies.

2.4. Statistical Analysis. The parameters reported in two or more studies were stratified based on the type of markers and sample source (serum or erythrocytes). Then, metaanalysis of the following biomarkers was conducted: total oxidant status (TOS), malondialdehyde (MDA), myeloperoxidase (MPO), advanced oxidation protein products (AOPP), nitric oxide (NO), total antioxidant status (TAS), paraoxonase $(\mathrm{PON})$, arylesterase (ARE), superoxide dismutase (SOD), catalase (CAT), glutathione peroxidase (GPx), thiol, and glutathione reductase (GR). Standardized mean difference (SMD) and 95\% confidence intervals (CIs) of oxidative and antioxidative markers were calculated. Median, range, or interquartile interval was transformed into mean and SD according to the method of Hozo et al. [17]. The heterogeneity among included studies was calculated using the $I^{2}$ statistic. The random effect model was adopted if the heterogeneity was high $\left(I^{2}>50 \%\right.$ or $\left.p<0.1\right)$; otherwise, fixed effect model was adopted. The publication bias was assessed by Egger's test. Sensitivity analysis was conducted for oxidative and antioxidative biomarkers measured in more than three studies by excluding a study one by one and repeating the meta-analysis. Statistical analyses were performed using STATA software (version 15.1, StataCorp, College Station, US). $p$ value $<0.05$ was considered statistically significant. 


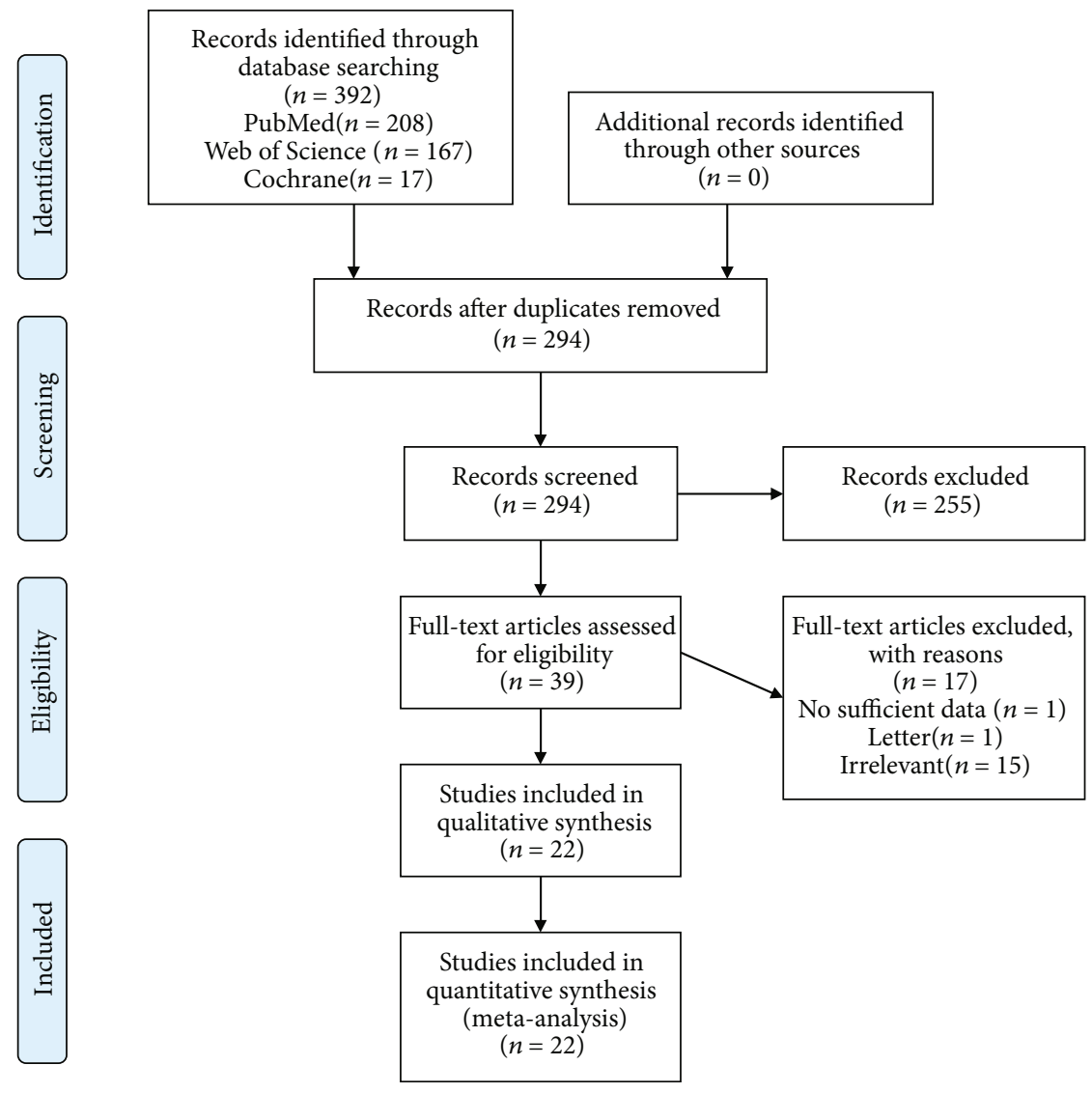

FIgURE 1: PRISMA flow chart of study selection.

\section{Results}

The study selection process was shown in Figure 1. Primary search generated 392 records, and 98 of duplications were removed. After screening the titles and abstracts, 255 articles were excluded. 39 full-text articles were assessed, and 17 articles were excluded because of data insufficiency and irrelevance of content or article type. Finally, 22 studies with 931 AS patients and 917 healthy controls were included in the systematic review and meta-analysis.

3.1. Characteristics of Studies. Table 1 shows the characteristics of the 22 included studies [3-5, 8, 9, 18-34]. Four studies $[8,9,25,33]$ measured the oxidative and (or) antioxidative biomarkers in serum and erythrocytes and the others in serum only. Six studies $[3,5,8,19,23,30]$ were included to analyze the association between oxidative stress and disease activity because they evaluated oxidative stress markers in patients with different disease activity. All studies ascertained patients using the modified New York criteria except two studies [4, 34]. One of them used the Assessment in SpondyloArthritis international Society (ASAS) criteria [4], and the other confirmed patients according to X-ray, MRI, CT, and laboratory inspections [34]. Part of patients in nine studies had taken nonsteroidal anti-inflammatory agent (NSAID), and no included patients used biological agents such as TNF- $\alpha$ blockers.
3.2. Meta-Analysis of Oxidative Stress Biomarkers. Table 2 summarizes the results of all meta-analyses in this study. Heterogeneity was high in the meta-analysis (all $I^{2}>50 \%$ ); therefore, the random-effect model was adopted. The forest plot of oxidative stress biomarkers in serum and erythrocytes of AS patients and controls is presented in Figure 2. The levels of overall oxidative stress markers in serum were significantly higher in AS patients $(\mathrm{SMD}=1.18 ; 95 \% \mathrm{CI} 0.85$ to $1.51, p<0.001)$. The concentrations of TOS (SMD $=1.08$; $95 \%$ CI 0.57 to $1.58, p<0.001)$, MDA (SMD $=1.71 ; 95 \% \mathrm{CI}$ 1.03 to $2.39, p<0.001)$, NO (SMD $=0.44 ; 95 \%$ CI 0.08 to $0.80, p=0.018)$, and AOPP (SMD $=2.19 ; 95 \%$ CI 1.02 to $3.37, p<0.001)$ were significantly higher in serum from AS patients compared with healthy controls. However, the levels of $\mathrm{MPO} \mathrm{SMD}=0.95 ; 95 \% \mathrm{CI}-0.64$ to $2.55, p=0.242)$ in serum and $\mathrm{MDA}(\mathrm{SMD}=0.71 ; 95 \% \mathrm{CI}-0.38$ to $1.79, p=$ 0.202 ) in erythrocytes showed nonsignificant difference between two groups.

The result of Egger's test for the included studies that measured oxidative stress markers in serum showed no significant publication bias $(p=0.084)$. Publication bias of MDA in erythrocytes was not examined due to small number of included studies. The levels of the above biomarkers remained similar in sensitivity analysis after removing studies one by one.

3.3. Meta-Analysis of Antioxidative Biomarkers. Heterogeneity was high in this meta-analysis (all $I^{2}>50 \%$ ); therefore, the 
TABLE 1: Characteristics of included studies.

\begin{tabular}{|c|c|c|c|c|c|}
\hline Study ID & Country & Case number $(\mathrm{M} / \mathrm{F})$ & Control number $(\mathrm{M} / \mathrm{F})$ & Biomarkers and main outcomes & NOS \\
\hline Reinisch et al. 1999 & Austria & $20(9 / 11)$ & $9(5 / 4)$ & Serum-TAS $\uparrow$ & 7 \\
\hline Ozgocmen et al. 2004 & Turkey & $15(12 / 3)$ & $16(13 / 3)$ & Serum-MDA $\uparrow, \mathrm{NO}^{\mathrm{NS}}, \mathrm{CAT} \uparrow, \mathrm{SOD}^{\mathrm{NS}}$ & 9 \\
\hline Yazici et al. 2004 & Turkey & $41(33 / 8)$ & $30(24 / 6)$ & Serum-AOPP $\uparrow$, MPO $\uparrow$, Thiol $\downarrow$ & 8 \\
\hline Karakoc et al. 2007 & Turkey & $50(42 / 8)$ & $26(20 / 6)$ & Serum-TOS $\uparrow, T A S \downarrow$ & 9 \\
\hline Erdem et al. 2010 & Turkey & $32(23 / 9)$ & $25(18 / 7)$ & Serum-PON1 ${ }^{\mathrm{NS}}, \mathrm{ARE}^{\mathrm{NS}}$ & 8 \\
\hline Karkucak et al. 2010 & Turkey & $24(4 / 20)$ & $27(5 / 22)$ & Serum-TOS ${ }^{\mathrm{NS}}$, TAS $^{\mathrm{NS}}$ & 9 \\
\hline Kozaci et al. 2010 & Turkey & $25(12 / 13)$ & $18(9 / 9)$ & Serum-MDA ${ }^{\mathrm{NS}}, \mathrm{NO}^{\mathrm{NS}}$ & 9 \\
\hline Stanek et al. 2010 & Poland & $16(16 / 0)$ & $16(16 / 0)$ & $\begin{array}{c}\text { Serum-MDA } \uparrow, \mathrm{SOD} \downarrow, \mathrm{TAS} \downarrow \\
\text { erythrocytes-MDA } \\
\text { GP }, \text { SOD } \downarrow, \mathrm{CAT}^{\mathrm{NS}}, \\
\end{array}$ & 8 \\
\hline Cece et al. 2011 & Turkey & $45(36 / 9)$ & $30(23 / 7)$ & Serum-PON1 $\downarrow, A R E \downarrow$ & 9 \\
\hline Nazıroğlu et al. 2011 & Turkey & $13(8 / 5)$ & $13(8 / 5)$ & $\begin{array}{c}\text { Serum-MDA } \downarrow, \mathrm{GSH}^{\mathrm{NS}} \\
\text { erythrocytes-MDA } \downarrow, \mathrm{GSH} \uparrow, \mathrm{GPx} \uparrow\end{array}$ & 9 \\
\hline Olama et al. 2013 & Egypt & $35(33 / 2)$ & $35(33 / 2)$ & Serum-PON1 $1, \mathrm{ARE} \downarrow, \mathrm{MDA} \uparrow$ & 9 \\
\hline Solmaz et al. 2013 & Turkey & $85(65 / 20)$ & $56(39 / 17)$ & Serum-TOS $\uparrow$, TAS $^{\text {NS }}$ & 9 \\
\hline Andrade et al. 2014 & Brazil & $14(11 / 3)$ & $26(16 / 10)$ & Serum-MPO $\uparrow, \mathrm{NO} \uparrow$ & 8 \\
\hline Liu et al. 2014 & China & $120(100 / 20)$ & $60(50 / 10)$ & $\begin{array}{c}\text { Serum-ROS } \uparrow, \mathrm{RNS} \uparrow, \mathrm{NO} \uparrow, \mathrm{MDA} \uparrow, \\
\mathrm{SOD} \downarrow, \mathrm{CAT} \downarrow, \mathrm{TAS} \downarrow\end{array}$ & 7 \\
\hline Wang et al. 2014 & China & $40(13 / 27)$ & $50(16 / 34)$ & Serum-MPO $\uparrow$ & 7 \\
\hline Wang et al. 2015 & China & $102(102 / 0)$ & $102(102 / 0)$ & $\begin{array}{c}\text { Serum-AOPP } \uparrow, \mathrm{MDA} \uparrow \\
\text { erythrocytes-SOD } \downarrow, \mathrm{CAT} \downarrow, \mathrm{GPx} \downarrow\end{array}$ & 9 \\
\hline Dogru et al. 2016 & Turkey & $69(43 / 26)$ & $60(37 / 23)$ & Serum-Thiol $\downarrow$, disulfide $\downarrow$ & 8 \\
\hline Türkön et al. 2016 & Turkey & $40(17 / 23)$ & $35(24 / 11)$ & Serum-MDA $\uparrow$, TOS $\uparrow, \mathrm{TAS}^{\mathrm{NS}}, \mathrm{SOD} \downarrow, \mathrm{GPx} \downarrow$ & 9 \\
\hline Kucuk et al. 2017 & Turkey & $60(45 / 15)$ & $54(32 / 22)$ & Serum - TOS $\uparrow$ & 9 \\
\hline Stanek et al. 2017 & Poland & $48(48 / 0)$ & $48(48 / 0)$ & $\begin{array}{c}\text { Serum-TOS } \uparrow, \mathrm{MDA} \uparrow, \mathrm{SOD} \uparrow \\
\text { Erythrocytes-SOD } \uparrow, \mathrm{CAT} \uparrow, \mathrm{GPx} \uparrow, \mathrm{GR} \uparrow, \mathrm{MDA} \uparrow\end{array}$ & 9 \\
\hline Xu et al. 2017 & China & $128(97 / 31)$ & $146(111 / 35)$ & Serum-PON1 $\downarrow$ & 9 \\
\hline Pishgah et al. 2020 & Iran & 16 & 35 & $\begin{array}{l}\text { Serum-NO } \uparrow, \mathrm{TOS}^{\mathrm{NS}}, \mathrm{SOD} \uparrow \\
\text { Erythrocytes-GPx} \\
\mathrm{NS}, \mathrm{CAT} \uparrow\end{array}$ & 9 \\
\hline
\end{tabular}

Legend: $\uparrow$ means the level of this marker is higher in AS patients compared with controls, and $\downarrow$ means the level of this marker is lower in AS patients compared with controls. NS means the level of this marker is not significantly different between AS patients and controls. NOS: Newcastle-Ottawa Scale; TAS: total antioxidant status; MDA: malondialdehyde; NO: nitric oxide; SOD: superoxide dismutase; AOPP: advanced oxidation protein products; MPO: myeloperoxidase; TOS: total oxidant status; PON1: paraoxonase 1; ARE: arylesterase; CAT: catalase; GPx: glutathione peroxidase; GR: glutathione reductase; GST: glutathione transferase; GSH: reduced glutathione; RNS: reactive nitrogen species. Oxidative stress linked markers are MDA, NO, AOPP, MPO, TOS, and RNS. Antioxidative stress linked markers are TAS, SOD, PON1, ARE, CAT, GPx, GR, GST, and GSH.

random-effect model was adopted. Figure 3 shows the levels of antioxidative stress biomarkers in serum and erythrocytes. The levels of overall antioxidative stress markers in serum were significantly lower in AS patients compared with controls $(\mathrm{SMD}=-0.76,95 \% \mathrm{CI}-1.17$ to $-0.36, p<0.001)$, while the levels in erythrocytes were not significantly different $(\mathrm{SMD}=-0.60,95 \% \mathrm{CI}-1.31$ to $0.11, p=0.100)$. The levels of TAS (SMD $=-1.19 ; 95 \%$ CI -2.35 to $-0.03, p=0.044$ ) and PON1 $(\mathrm{SMD}=-1.01 ; 95 \% \mathrm{CI}-1.78$ to $-0.24, p=0.010)$ were lower in AS patients. The results of other biomarkers in serum and all antioxidative stress markers in erythrocytes were not significant. The result of sensitivity analysis remained essentially unchanged in both serum and erythrocytes. Publication bias was not assessed due to small number of included studies.
3.4. Meta-Analysis of Oxidative Biomarkers in Serum of Patients with Different Disease Activity. Six studies reported the levels of oxidative biomarkers in patients with different disease activity. Patients with the Bath Ankylosing Spondylitis Disease Activity Index (BASDAI) $\geq 4$ were classified into the active group, and BASDAI $<4$ were classified into the inactive group. Heterogeneity in each biomarker subgroup was low and statistically nonsignificant (MDA: $I^{2}=0, p=$ 0.627; AOPP: $I^{2}=0, p=0.964$; NO: $\left.I^{2}=0, p=0.703\right)$. Therefore, the fixed effect model was used. The overall levels of oxidative markers in serum were significantly higher in active patients compared with inactive patients $(\mathrm{SMD}=0.42 ; 95 \%$ CI 0.02 to $0.80, p<0.001$ ) (Figure 4 ). The levels of MDA $(\mathrm{SMD}=0.51 ; 95 \% \mathrm{CI} 0.21$ to $0.81, p=0.001)$ and AOPP $(\mathrm{SMD}=0.95 ; 95 \%$ CI 0.58 to $1.31, p<0.001)$ were 
TABLE 2: Results of meta-analyses.

\begin{tabular}{|c|c|c|c|c|c|c|c|}
\hline & & Markers & Study N & Model effect & SMD (95\% CI) & $I^{2}$ & $p$ value \\
\hline \multirow{16}{*}{ Compared with healthy controls } & \multirow{5}{*}{$\begin{array}{l}\text { Oxidative stress markers } \\
\text { in serum }\end{array}$} & TOS & 7 & Random & $1.03(0.49,1.57)$ & $88.70 \%$ & $<0.001$ \\
\hline & & MDA & 10 & Random & $1.71(1.03,2.39)$ & $93.50 \%$ & $<0.001$ \\
\hline & & MPO & 3 & Random & $0.95(-0.64,2.55)$ & $95.70 \%$ & 0.242 \\
\hline & & NO & 6 & Random & $0.44(0.08,0.80)$ & $57.40 \%$ & 0.018 \\
\hline & & AOPP & 2 & Random & $2.19(1.02,3.37)$ & $90.10 \%$ & $<0.001$ \\
\hline & \multirow[t]{2}{*}{$\begin{array}{l}\text { Oxidative stress markers } \\
\text { in erythrocytes }\end{array}$} & MDA & 3 & Random & $0.71(-0.38,1.79)$ & $87.40 \%$ & 0.202 \\
\hline & & TAS & 7 & Random & $-1.19(-2.35,-0.03)$ & $96.80 \%$ & 0.044 \\
\hline & \multirow{5}{*}{$\begin{array}{l}\text { Antioxidative stress } \\
\text { markers in serum }\end{array}$} & PON 1 & 4 & Random & $-1.01(-1.78,-0.24)$ & $91.70 \%$ & 0.010 \\
\hline & & ARE & 3 & Random & $-1.32(-2.78,0.15)$ & $95.20 \%$ & 0.078 \\
\hline & & CAT & 3 & Random & $0.10(-0.82,1.03)$ & $87.00 \%$ & 0.830 \\
\hline & & SOD & 7 & Random & $-0.34(-1.21,0.53)$ & $94.50 \%$ & 0.437 \\
\hline & & Thiol & 2 & Random & $-0.75(-1.78,0.26)$ & $90.90 \%$ & 0.154 \\
\hline & \multirow{4}{*}{$\begin{array}{l}\text { Antioxidative stress markers } \\
\text { in erythrocytes }\end{array}$} & CAT & 4 & Random & $0.14(-0.91,1.20)$ & $95.20 \%$ & 0.791 \\
\hline & & GPx & 5 & Random & $-0.64(-1.50,0.22)$ & $92.70 \%$ & 0.144 \\
\hline & & SOD & 3 & Random & $-1.85(-5.12,1.43)$ & $99.10 \%$ & 0.268 \\
\hline & & GR & 2 & Random & $-0.07(-1.37,1.24)$ & $90.00 \%$ & 0.921 \\
\hline \multirow{3}{*}{ Compared with inactive AS patients } & \multirow{3}{*}{$\begin{array}{l}\text { Oxidative stress markers } \\
\text { in serum of patients with } \\
\text { different disease activity }\end{array}$} & MDA & 4 & Fixed & $0.51(0.21,0.81)$ & $0.00 \%$ & 0.001 \\
\hline & & AOPP & 2 & Fixed & $0.95(0.58,1.31)$ & $0.00 \%$ & $<0.001$ \\
\hline & & $\mathrm{NO}$ & 2 & Fixed & $-0.52(-1.06,0.02)$ & $0.00 \%$ & 0.061 \\
\hline
\end{tabular}

Legend: TOS: total oxidant status; MDA: malondialdehyde; NO: nitric oxide; AOPP: advanced oxidation protein products; MPO: myeloperoxidase; TAS: total antioxidant status; SOD: superoxide dismutase; PON1: paraoxonase 1; ARE: arylesterase; CAT: catalase; GPx: glutathione peroxidase; GR: glutathione reductase.

significantly higher in active patients. The concentration of NO (SMD $=-0.52 ; 95 \%$ CI -1.06 to $0.02, p=0.061)$ showed no statically significant difference between the active and inactive group. Sensitivity analysis also revealed similar results in oxidative biomarkers by removing a study at a time. Publication bias was not assessed due to small number of studies.

\section{Discussion}

This meta-analysis including 22 studies with comparisons of oxidative and antioxidative stress markers between AS patients and healthy controls revealed that most of oxidative biomarkers in serum including TOS, MDA, AOPP, and NO were higher in AS patients. Additionally, the antioxidative markers such as TAS and PON1 were significantly lower in AS patients. Besides, we also found that the levels of AOPP and MDA were higher in AS patients with higher disease activity.

Following theories may explain why oxidative biomarkers in serum were higher in AS patients and the role of oxidative stress in the pathogenesis of AS. Firstly, cell membranes, lipids, nucleic acids, proteins, and extracellular matrix can be damaged by oxidative stress, leading to an increased level of oxidative injury products [31]. ROS which were highly produced in oxidative stress are also highly reactive species with one or more uncoupled electrons, increasing lipid peroxidation and protein oxidation [35]. At the site of inflammation in AS, the level of activated leukocytes increases, leading to the release of ROS and enzymes such as MPO, which can induce the formation of hypochlorous acid [3]. Hypochlorous acid causes oxidation of proteins and tissue injury by reacting with the components of cartilage [29]. Secondly, lipid peroxidation influences the fluidity and receptor functions of cell membranes by reacting with cell membranes and inducing phospholipase A2 [25]. As a result, $\mathrm{T}$ cells can be induced to secret more interleukins to increase inflammation and lipid peroxidation, causing a vicious circle [25]. MDA and AOPP, the products of lipid and protein oxidation, respectively, can act as secondary mediators to cause further damage and increase disease progression by activating the TNF- $\alpha$ and NF- $\kappa \mathrm{B}$ pathways, which play essential roles in the inflammatory process of AS [8]. However, there was no significant difference of MDA in erythrocytes between patients and controls. One of the possible explanations is that erythrocyte membranes can react with MDA, causing the increased permeability of erythrocyte membranes for different substances and penetration of MDA from erythrocyte to serum [9]. Therefore, the level of MDA in serum may be more reliable than that in erythrocytes.

The antioxidant systems against toxic activity of ROS by transferring electrons to oxidants include enzymatic system such as GPx, SOD, PON1, and CAT and non-enzymatic system such as victim $A, C$, and $E$, which can be indicated by the total antioxidant status (TAS) in serum $[9,36]$. Our study demonstrated a significantly lower level of TAS in serum from AS patients, which may reveal an increased depletion of antioxidant systems in serum due to the increased 
Oxidative stress markers in serum

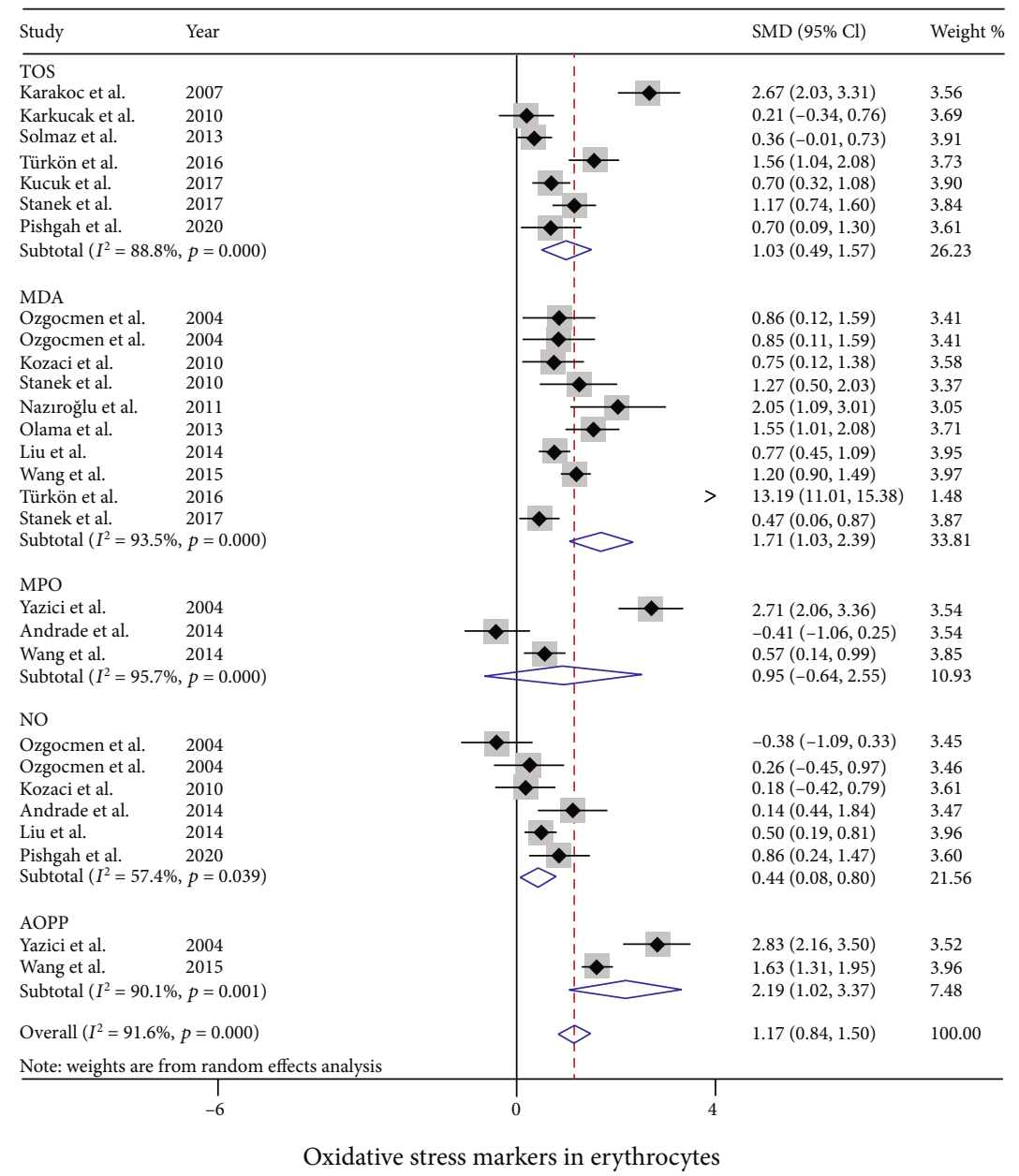

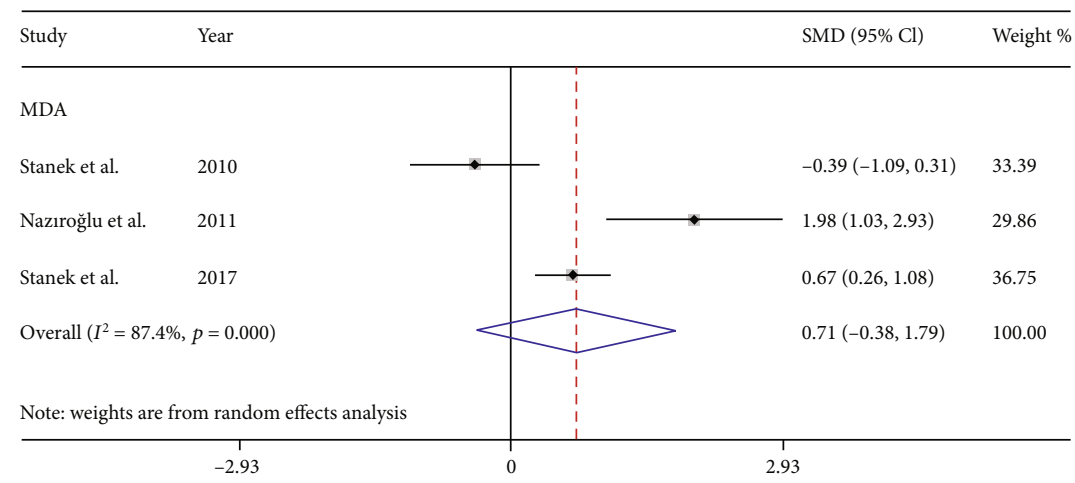

FIgURE 2: Forest plot of oxidative stress markers in serum and erythrocytes.

production of ROS. PON1 produced by the liver is an important antioxidative enzyme in endogenous free radical elimination and is also related to high density lipoprotein (HDL) [5]. The decreased concentration of PON1 shown in this study may indicate the increased consumption of antioxidative enzyme systems influenced by the increased inflammation and oxidative stress. Considering its antiatherogenic properties, the decreased level of PON 1 may increase the risk of cardiovascular disease of AS patients. Cece et al. have found that the decreased PON 1 activity is associated with the increased carotid intima-media thickness [24]. However, the levels of other antioxidants such as SOD and CAT were not significantly different between AS patients and controls. The levels of SOD in AS patients were inconsistent in different studies. Based on current literatures, increased SOD level could be a protective response of the antioxidative system against the harmful effect of oxidative stress while the decreased SOD level may due to the consumption of antioxidant $[8,19]$. Evidence suggests that CAT may not be a reliable marker of antioxidative stress [9]. Only three studies in this meta-analysis measured the level of CAT, and the results may not be reliable enough due to the small sample size. In 
Antioxidative stress markers in serum

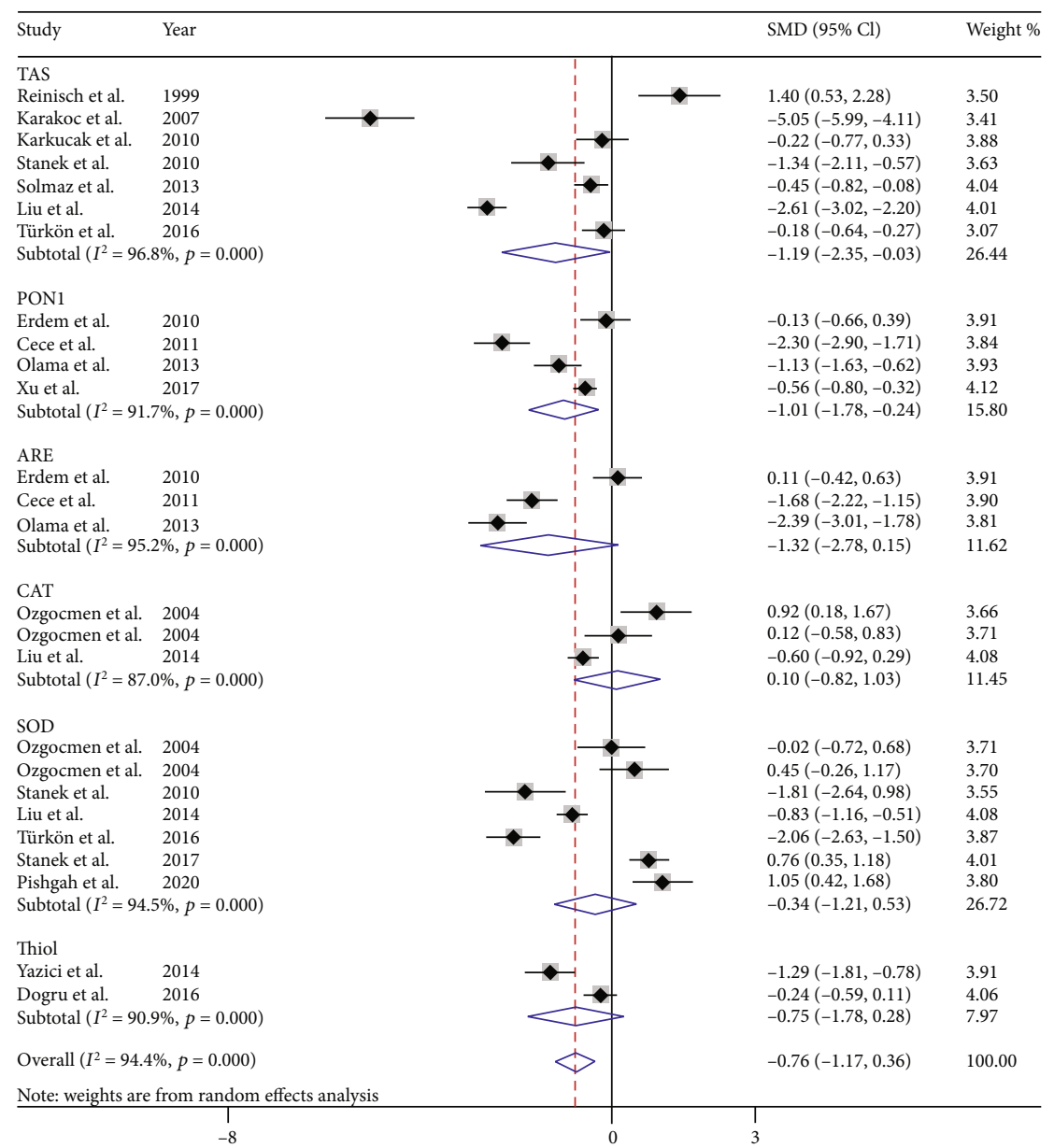

Antioxidative stress markers in erythrocytes

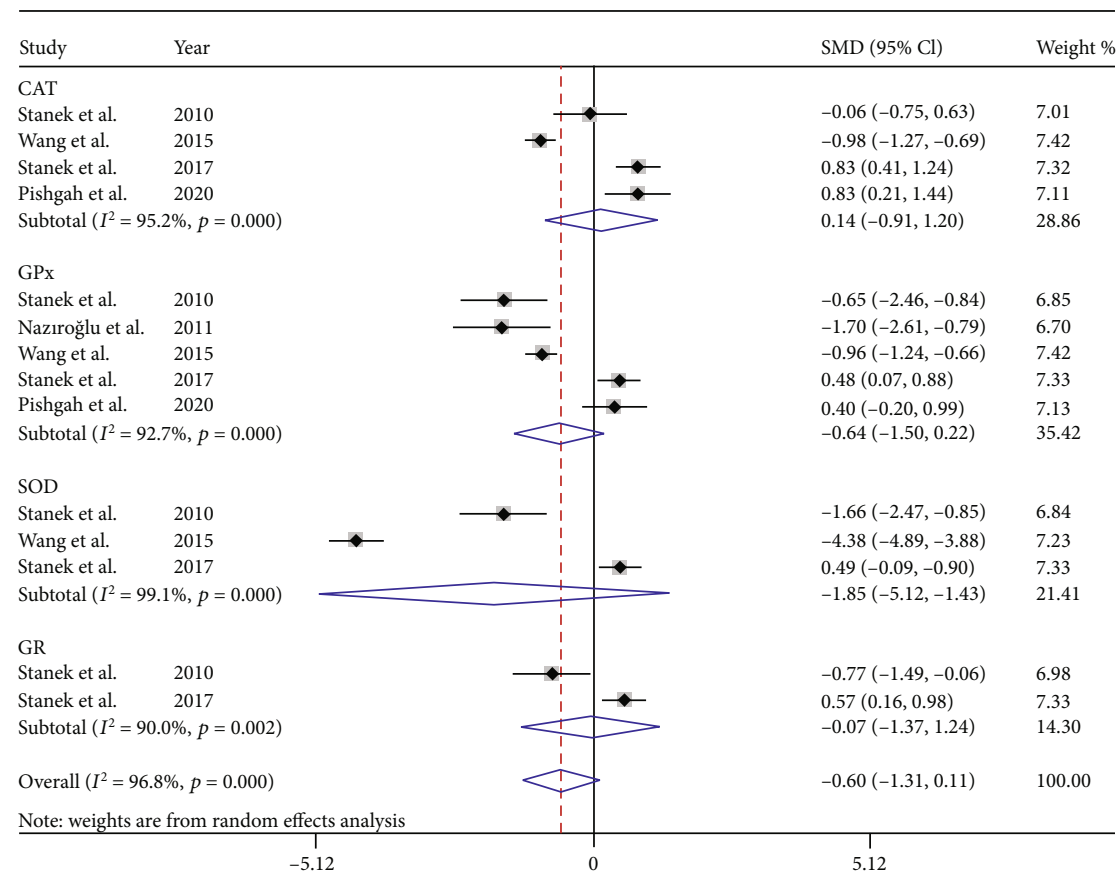

FIGURE 3: Forest plot of antioxidative stress markers in serum and erythrocytes. 
Antioxidative stress markers in different disease activity

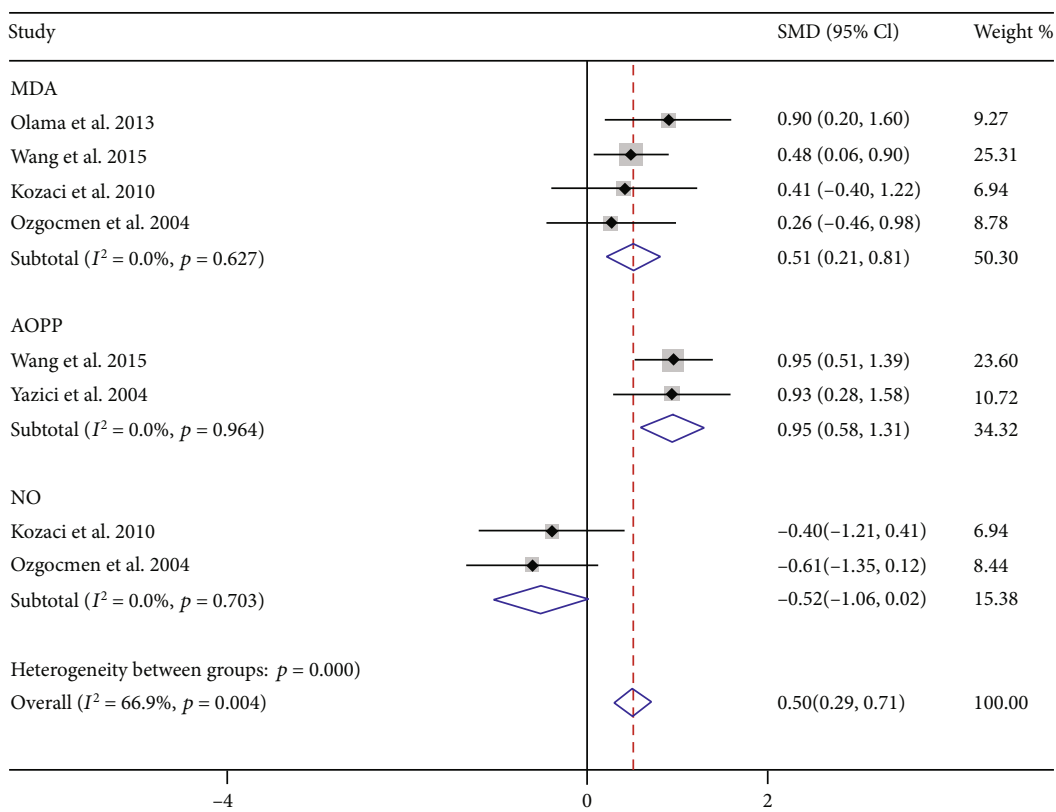

FIGURE 4: Forest plot of oxidative stress markers in serum of patients with different disease activity.

conclusion, the change of the antioxidant level in AS needs to be clarified in future studies.

According to the scores of the Bath Ankylosing Spondylitis Disease Activity Index (BASDAI) used for patients and the visual analogue scale (VAS) used for physician, and the levels of CRP and ESR, AS patients can be divided into two groups: active patients with severe symptoms and inactive patients with mild symptoms. In this study, six articles evaluated the oxidative and/or antioxidative stress markers in AS patients with different disease activity $[3,5,8,19,23,30]$. The result of meta-analysis showed that the level of MDA and AOPP was higher in active patients, indicating a close relationship between oxidative injury products and the disease activity of AS. Therefore, the oxidative stress biomarkers are expected to be the biomarkers reflecting the disease activity of AS. However, we failed to analyze the association between antioxidative stress markers and the disease activity of AS because of the limited number of studies assessing the antioxidants between active and inactive AS patients.

Our study has several limitations. Firstly, the heterogeneity between the included studies is high, which might be due to different participants, sample sizes, disease activities, and measuring methods of different studies. Although the inclusion and exclusion criteria for patients and healthy controls in different studies were similar, the participants were still not homogenous in different studies considering the effect of race and sex ratio. Secondly, the small sample sizes in some studies may have an influence on our results as well. Thirdly, the limited data restrict the conduction of meta-regression analysis. Last but not least, we also found a relationship between the level of oxidative stress markers and disease activity, but the number of included studies is small. Further investigations are needed to explain the role of oxidative stress in AS.

\section{Conclusions}

This meta-analysis demonstrated an overall increase of oxidative markers and decrease of antioxidative markers in AS. Some oxidative biomarkers in serum such as AOPP and MDA are associated with the disease activity. In conclusion, this study suggests that oxidative stress linked markers may serve as novel biomarkers in AS patients. In addition, oxidative stress may play an important role in the pathogenesis of AS and may be a potential therapeutic target for AS.

\section{Data Availability}

The data supporting this systematic review and meta-anaysis are from previously reported studies, which have been cited. The processed data are available from the corresponding author upon request.

\section{Conflicts of Interest}

No conflicts of interest.

\section{Authors' Contributions}

Jiaxiao Li and Suling Liu contributed equally to this work and should be considered as co-first authors.

\section{Acknowledgments}

Science and technology general project of Guangzhou, China; funding number: 201804010028. 


\section{References}

[1] J. Sieper and D. Poddubnyy, "Axial spondyloarthritis," Lancet, vol. 390, no. 10089, pp. 73-84, 2017.

[2] J. A. Smith, "Update on ankylosing spondylitis: current concepts in pathogenesis," Current Allergy and Asthma Reports, vol. 15, no. 1, p. 489, 2015.

[3] C. Yazici, K. Köse, M. Calis, S. Kuzugüden, and M. Kirnap, "Protein oxidation status in patients with ankylosing spondylitis," Rheumatology (Oxford), vol. 43, no. 10, pp. 1235-1239, 2004.

[4] A. Pishgahi, R. Abolhasan, S. Danaii et al., "Immunological and oxidative stress biomarkers in ankylosing spondylitis patients with or without metabolic syndrome," Cytokine, vol. 128, p. 155002, 2020.

[5] S. M. Olama and M. M. Elarman, "Evaluation of paraoxonase and arylesterase activities in Egyptian patients with ankylosing spondylitis," Rheumatology International, vol. 33, no. 6, pp. 1487-1494, 2013.

[6] S. Ezzat, M. L. Louka, Z. M. Zakaria, M. M. Nagaty, and R. G. Metwaly, "Autophagy in osteoporosis: relation to oxidative stress," Journal of Cellular Biochemistry, vol. 120, no. 2, pp. 2560-2568, 2018.

[7] R. D. Damavandi, S. N. Mousavi, F. Shidfar et al., "Effects of daily consumption of cashews on oxidative stress and atherogenic indices in patients with type 2 diabetes: a randomized, controlled-feeding trial," International Journal of Endocrinology and Metabolism, vol. In Press, no. In Press, 2019.

[8] L. Wang, L. Gao, D. Jin et al., “The relationship of bone mineral density to oxidant/antioxidant status and inflammatory and bone turnover markers in a multicenter cross-sectional study of young men with ankylosing spondylitis," Calcified Tissue International, vol. 97, no. 1, pp. 12-22, 2015.

[9] A. Stanek, G. Cieślar, E. Romuk et al., "Decrease in antioxidant status of plasma and erythrocytes from patients with ankylosing spondylitis," Clinical Biochemistry, vol. 43, no. 6, pp. 566570, 2010.

[10] K. Liu, L. Wu, X. Shi, and F. Wu, "Protective effect of naringin against ankylosing spondylitis via ossification, inflammation and oxidative stress in mice," Experimental and Therapeutic Medicine, vol. 12, no. 2, pp. 1153-1158, 2016.

[11] F. Farsi, J. Heshmati, A. Keshtkar et al., "Can coenzyme Q10 supplementation effectively reduce human tumor necrosis factor- $\alpha$ and interleukin-6 levels in chronic inflammatory diseases? A systematic review and meta-analysis of randomized controlled trials," Pharmacological Research, vol. 148, article 104290, 2019.

[12] M. W. Miller, A. P. Lin, E. J. Wolf, and D. R. Miller, "Oxidative stress, inflammation, and Neuroprogression in chronic PTSD," Harvard Review of Psychiatry, vol. 26, no. 2, pp. 5769, 2018

[13] A. R. Phull, B. Nasir, I. . Haq, and S. J. Kim, "Oxidative stress, consequences and ROS mediated cellular signaling in rheumatoid arthritis," Chemico-Biological Interactions, vol. 281, pp. 121-136, 2018.

[14] M. N. Magrey and M. A. Khan, "The paradox of bone formation and bone loss in ankylosing spondylitis: evolving new concepts of bone formation and future trends in management," Current Rheumatology Reports, vol. 19, no. 4, 2017.

[15] A. R. G. Susperregui, C. Gamell, E. Rodríguez-Carballo et al., "Noncanonical BMP signaling regulates cyclooxygenase-2 transcription," Molecular Endocrinology, vol. 25, no. 6, pp. 1006-1017, 2011.

[16] D. Y. Zhang, Y. Pan, C. Zhang et al., "Wnt/ $\beta$-catenin signaling induces the aging of mesenchymal stem cells through promoting the ROS production," Molecular and Cellular Biochemistry, vol. 374, no. 1-2, pp. 13-20, 2013.

[17] S. P. Hozo, B. Djulbegovic, and I. Hozo, "Estimating the mean and variance from the median, range, and the size of a sample," BMC Medical Research Methodology, vol. 5, no. 1, 2005.

[18] N. Reinisch, E. Mur, M. Herold et al., "Decrease of respiratory burst in neutrophils of patients with ankylosing spondylitis by combined radon-hyperthermia treatment," Clinical and Experimental Rheumatology, vol. 17, no. 3, pp. 335-338, 1999.

[19] S. Ozgocmen, S. Sogut, O. Ardicoglu, E. Fadillioglu, I. Pekkutucu, and O. Akyol, "Serum nitric oxide, catalase, superoxide dismutase, and malondialdehyde status in patients with ankylosing spondylitis," Rheumatology International, vol. 24 , no. 2, pp. 80-83, 2004.

[20] M. Karakoc, O. Altindag, H. Keles, N. Soran, and S. Selek, "Serum oxidative-antioxidative status in patients with ankylosing spondilitis," Rheumatology International, vol. 27, no. 12, pp. 1131-1134, 2007.

[21] F. H. Erdem, S. Karatay, K. Yildirim, and A. Kiziltunc, "Evaluation of serum paraoxonase and arylesterase activities in ankylosing spondylitis patients," Clinics (São Paulo, Brazil), vol. 65, no. 2, pp. 175-179, 2010.

[22] M. Karkucak, E. Capkin, A. Alver et al., "The effect of antiTNF agent on oxidation status in patients with ankylosing spondylitis," Clinical Rheumatology, vol. 29, no. 3, pp. 303307,2010

[23] L. D. Kozaci, I. Sari, A. Alacacioglu, S. Akar, and N. Akkoc, "Evaluation of inflammation and oxidative stress in ankylosing spondylitis: a role for macrophage migration inhibitory factor," Modern Rheumatology, vol. 20, no. 1, pp. 34-39, 2010.

[24] H. Cece, P. Yazgan, E. Karakas et al., "Carotid intima-media thickness and paraoxonase activity in patients with ankylosing spondylitis," Clinical and Investigative Medicine, vol. 34, no. 4, article E225, 2011.

[25] M. Nazıroğlu, S. Akkuş, and H. Celik, "Levels of lipid peroxidation and antioxidant vitamins in plasma and erythrocytes of patients with ankylosing spondylitis," Clinical Biochemistry, vol. 44, no. 17-18, pp. 1412-1415, 2011.

[26] D. Solmaz, D. Kozac1, I. Sari et al., "AB0532 Oxidative stress and its related factors in patients with ankylosing spondylitis," Annals of the Rheumatic Diseases, vol. 72, Suppl 3, pp. A952.1A9A952, 2014.

[27] K. R. de Andrade, G. R. W. de Castro, G. Vicente et al., "Evaluation of circulating levels of inflammatory and bone formation markers in axial spondyloarthritis," International Immunopharmacology, vol. 21, no. 2, pp. 481-486, 2014.

[28] J. Liu, Y. Qi, L. Zheng et al., "Xinfeng capsule improves pulmonary function in ankylosing spondylitis patients via NF- KBiNOS-NO signaling pathway," Journal of traditional chinese medicine $=$ chung $i$ tsa chih ying wen pan, vol. 34, no. 6, pp. 657-665, 2014.

[29] W. Wang, Z. Jian, J. Guo, and X. Ning, "Increased levels of serum myeloperoxidase in patients with active rheumatoid arthritis," Life Sciences, vol. 117, no. 1, pp. 19-23, 2014.

[30] A. Dogru, A. Balkarli, G. Y. Cetin et al., "Thiol/disulfide homeostasis in patients with ankylosing spondylitis," Bosnian 
Journal of Basic Medical Sciences, vol. 16, no. 3, pp. 187-192, 2016.

[31] H. Türkön, F. Gökmen, D. Ü. Çakir et al., "Increased levels of serum ischemia modified albumin in patients with ankylosing spondylitis," Clinical Laboratory, vol. 62, no. 4, pp. 645-649, 2016.

[32] A. Kucuk, A. Uğur Uslu, A. Icli et al., "The LDL/HDL ratio and atherosclerosis in ankylosing spondylitis," Zeitschrift für Rheumatologie, vol. 76, no. 1, pp. 58-63, 2017.

[33] A. Stanek, A. Cholewka, T. Wielkoszyński, E. Romuk, K. Sieroń, and A. Sieroń, "Increased levels of oxidative stress markers, soluble CD40 ligand, and carotid intima-media thickness reflect acceleration of atherosclerosis in male patients with ankylosing spondylitis in active phase and without the classical cardiovascular risk factors," Oxidative Medicine and Cellular Longevity, vol. 2017, Article ID 9712536, 8 pages, 2017.

[34] H. Xu and Y. Qu, "Correlation of PON1 polymorphisms with ankylosing spondylitis susceptibility," Medicine, vol. 96, no. 42, p. e7416, 2017.

[35] M. Sepidarkish, M. Akbari-Fakhrabadi, E. Daneshzad et al., "Effect of omega-3 fatty acid plus vitamin E cosupplementation on oxidative stress parameters: a systematic review and meta-analysis," Clinical Nutrition, vol. 39, no. 4, pp. 1019-1025, 2020.

[36] S. Fazelian, M. Hoseini, N. Namazi et al., "Effects of L-arginine supplementation on antioxidant status and body composition in obese patients with pre-diabetes: a randomized controlled clinical trial," Adv Pharm Bull, vol. 4, Suppl 1, pp. 449-454, 2014. 